\title{
Extended-spectrum Beta-lactamase Positive Klebsiella pneumoniae
}

National Cancer Institute

\section{Source}

National Cancer Institute. Extended-spectrum Beta-lactamase Positive Klebsiella pneumoniae. NCI Thesaurus. Code C139101.

A subgroup of Klebsiella pneumoniae bacteria that produce beta-lactamase and are therefore resistant to extended-spectrum beta-lactam (penicillin) antibiotics. 